\title{
Gaseous flows in galaxies
}

\author{
Francoise Combes ${ }^{1}$ \\ ${ }^{1}$ Observatoire de Paris, LERMA (CNRS:UMR8112), 61 Av. de l'Observatoire, \\ 75014 Paris, France \\ email: francoise.combes@obspm.fr
}

\begin{abstract}
The gas component plays a major role in the dynamics of spiral galaxies, because of its dissipative character, and its ability to exchange angular momentum with stars in the disk. Due to its small velocity dispersion, it triggers gravitational instabilities, and the corresponding non-axisymmetric patterns produce gravity torques, which mediate these angular momentum exchanges. When a srong bar pattern develops with the same pattern speed all over the disk, only gas inside corotation can flow towards the center. But strong bars are not long lived in presence of gas, and multiple-speed spiral patterns can develop between bar phases, and help the galaxy to accrete external gas flowing from cosmic filaments. The gas is then intermittently driven to the galaxy center, to form nuclear starbursts and fuel an active nucleus. The various time-scales of these gaseous flows are described.
\end{abstract}

Keywords. galaxies: spiral - galaxies: kinematics and dynamics - galaxies: interstellar matter - galaxies: bulges - galaxies: nuclei - galaxies: starburst - galaxies: interactions

\section{Evidence of gas flows and secular evolution}

A large wealth of data has recently accumulated, showing the importance of gas flow in the center, as part of secular evolution of galaxies. The comparison between the stellar profiles at $3.6 \mu \mathrm{m}$ and the dust profiles at $8 \mu \mathrm{m}$ with Spitzer, reveals that barred galaxies have an excess of gas mass towards the center, with respect to the extrapolation of the exponential profile (Regan et al. 2006). When the PAH emission is taken as a tracer of star formation rate, clear differences are revealed in the star formation modes between pseudobulges, identified by nuclear spirals or bars and flattening of the bulge, and classical bulge galaxies, with round isophotes. The latter possess star-forming outer disk, with a decline in star formation rates toward the center of the galaxy. Star formation is more spectacular in the center of pseudo-bulges $(\mathrm{r}<1.5 \mathrm{kpc}$, Fisher 2006). All galaxies with a central hole in their PAH $(8 \mu \mathrm{m})$ distribution are unbarred. This is expected since, without significant gas inflow, the central region of an exponential disk should consume its gas fuel much faster than the outer parts, according to the non-linear Schmidt law.

It was already established through $\mathrm{CO}$ emission that barred galaxies had enhanced central molecular gas concentrations, with respect to unbarred galaxies. (Sakamoto et al. 1999, Sheth et al. 2005), Now the observation of the same phenomenon in dust PAH emission at 8 microns consolidates these previous findings, and in particular indicates that this is not only due to an enhanced CO-to- $\mathrm{H}_{2}$ conversion ratio.

Another obvious evidence of gas flows is the existence if resonant rings in barred galaxies, where the gas is piling up, and maintained there by the gravity torques of the bar (e.g. Buta \& Combes 1996). The nature of these rings and their indication of the bar pattern speed is clear in galaxies with multiple-rings, corresponding to the inner or outer resonances. The PAH dust emission traces beautifully these rings, previously highlighted by star formation hot spots, or concentration of $\mathrm{CO}$ emission. 


\section{Angular momentum transfers}

Originally, the angular momentum $(\mathrm{AM})$ is created by tidal torques between structures at early times, before turn-around. The amount of AM (or J) produced can be quantified by the dimensionless quantity: $\lambda=J|E|^{1 / 2} / G M^{5 / 2} \sim 0.035$, where $\mathrm{E}$ is the energy and $\mathrm{M}$ the mass. At this stage, baryons and dark matter behave similarly, and they get the same specific angular momentum. But after turn-around, baryons dissipate and collapse, deeper inside dark haloes, and they lose AM at the benefit of the dark matter.

In simulation of galaxy mergers, AM is exchanged from baryons to the dark haloes, through tidal forces and dynamical friction. The visible component have then not enough $\mathrm{AM}$ to account for the observations. The dark haloes of merger remnants do not accumulate angular momentum: the gravitational heating due to the merger produces mass loss of the dark matter halo through the virial radius (D'Onghia \& Navarro 2007).

External gas infall could help to solve the AM problem of the standard CDM scenario. Only diffuse cold accretion after the last merger can reform an extended disk, and this accretion produces secular evolution, through gas flows.

In purely stellar disks, the secular evolution also exchanges AM from baryons to dark haloes; this is a way to enhance the formation of bars (Athanassoula 2002, 2003). If the $\mathrm{AM}$ transfer to the dark matter is saturated, due to an already rotating halo, AM is exchanged with the outer disk. The actual mode of transfer depends on the amount of dark matter present inside the visible disk. The AM exchange with the dark matter is made through resonances, the maximum efficiency being at corotation. When a bar is present in the disk, its pattern speed continuously decreases with time, due to dynamical friction on the dak halo, which means that the corotation resonance moves, and involves always new particles, that are progressively heated (Debattista \& Sellwood 2000).

When the galaxy is rich in gas, the angular momentum exchange is preferentially done between stars and gas, given the strong torques exerted from the non axisymmetric stellar patterns to the dissipative and density-contrasted gas component. Viscous torques are also present at a very low level, but are easily dominated by the gravity torques (Bournaud \& Combes 2002). Gravity torques change sign at each resonance, they drive the gas inwards from corotation to the center. In this radial gas inflow, the AM lost by the gas is given to the bar. It is comparable in amplitude to the AM of the bar wave, and contributes to weaken or destroy it. This rate of exchange has been quantified by observations: torques can be computed with the help of the near infrared images, as tracer of the mass distribution, and with the gas distribution $(\mathrm{H} \alpha, \mathrm{CO}, \mathrm{HI})$. The torque is proportional to the phase shift between the stellar bar and gas response, and the leading dust lanes characteristics of bars are already an obvious smoking gun (e.g. Garcia-Burillo et al. 2005).

The gravity torques are positive outside corotation, and the gas is flowing outwards until the outer Lindblad resonance (OLR). Does it mean that gas in the outer disks encounter difficulties to flow in? This might be the case when the bar is strong, and forces its pattern speed to the outer spiral. But in the case of a weak pattern, simulations show that the most frequent dynamical state of a galaxy is with multiple pattern speeds. The outer disk decouples from the center, and develops a spiral at lower pattern speed. This could be the case of many extended gaseous disks, which correspond sometimes to extended UV disks. The activity in the outer parts could be triggered by companions. An example is given by NGC 7479 in Fig. 1, where the atomic gas has a morphology apparently decoupled from the thin central bar. 


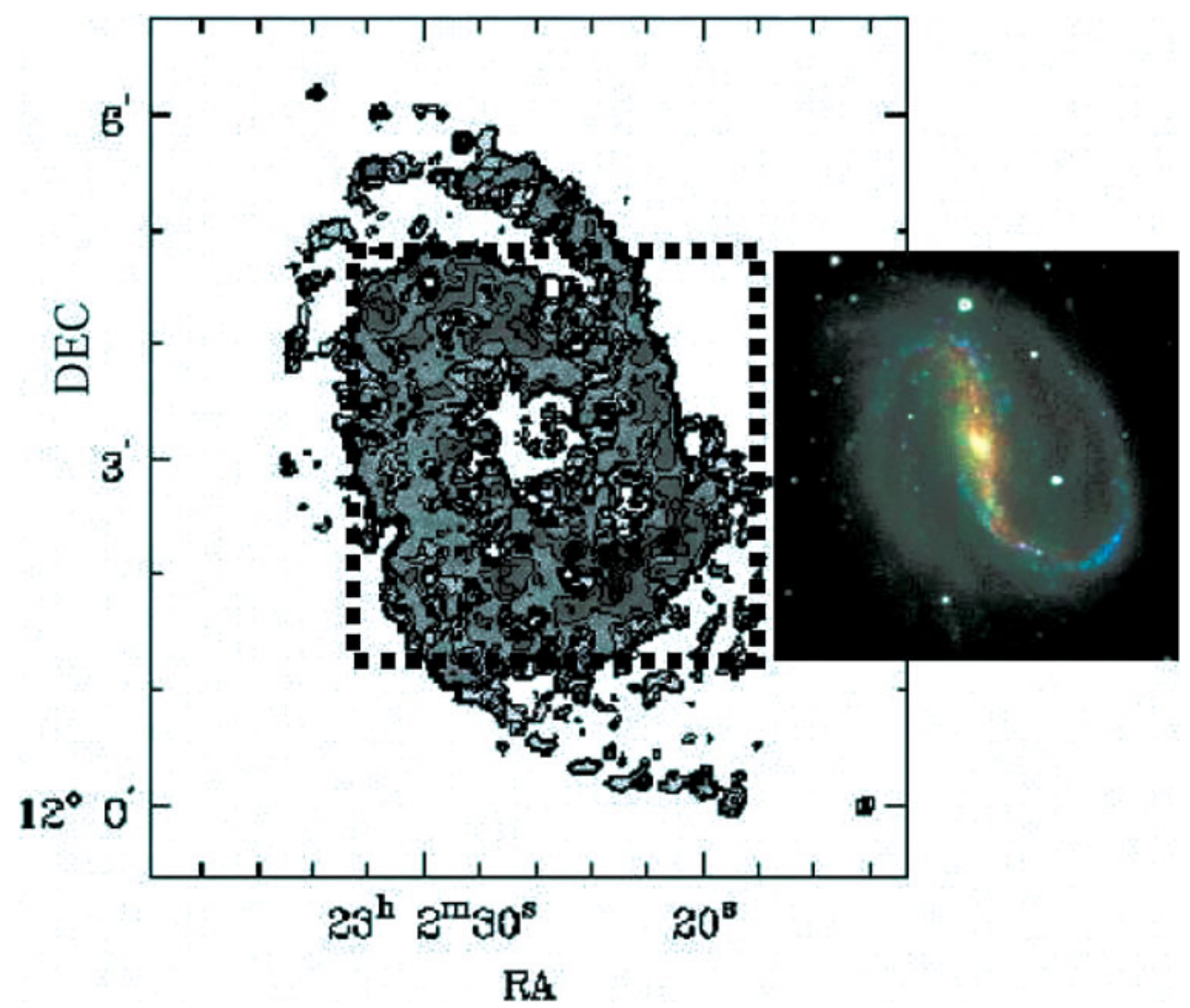

Figure 1. The two spiral patterns in NGC 7479: at the same scale, are compared an optical image, which should be overlaid in the dashed square, and the HI map from Laine et al. (1998a). Note that the optical spiral arms, getting out of the central bar, are winding up into a pseudo-ring, which is an outer Lindblad resonance (OLR). The inner structure is devoid of atomic gas, while it is rich in molecular gas, aligned along the bar (Laine et al. 1999). The HI emission begins in a ring, at the OLR of the optical pattern, and then continues in an external spiral structure.

The examination of the gravitational potential and rotation curve of NGC 7479 reveals that the pseudo-ring in the optical (and HI) might be the OLR of the central bar, and the ILR of the outer spiral pattern, as shown in Fig. 2.

\section{Bar destruction, role of gas and dark matter}

For more than a decade now, numerical simulations of barred galaxies with gas have revealed the fragility of bars, which can be easily detroyed by secular evolution (e.g. Friedli \& Benz 1993). But this destruction depends on several factors, and some controversy has been discussed in the literature. Since the gas inflow towards the center is accompanied by a central mass concentration, the latter has been first thought to tbe the main destruction mechanism. But a central mass concentration alone must be very high to succeed to weaken the bar (Shen \& Sellwood 2004, Athanassoula et al. 2005).

The main destruction mechanism could be the gas inflow itself, which is driven in by the bar torques. The angular momentum is taken up by the bar wave (e.g. Bournaud et al. 2005a). When the galactic disk is not dominated by dark matter, the AM exchange available for the stellar component is only the outer disk or the gas. The outer disk takes 


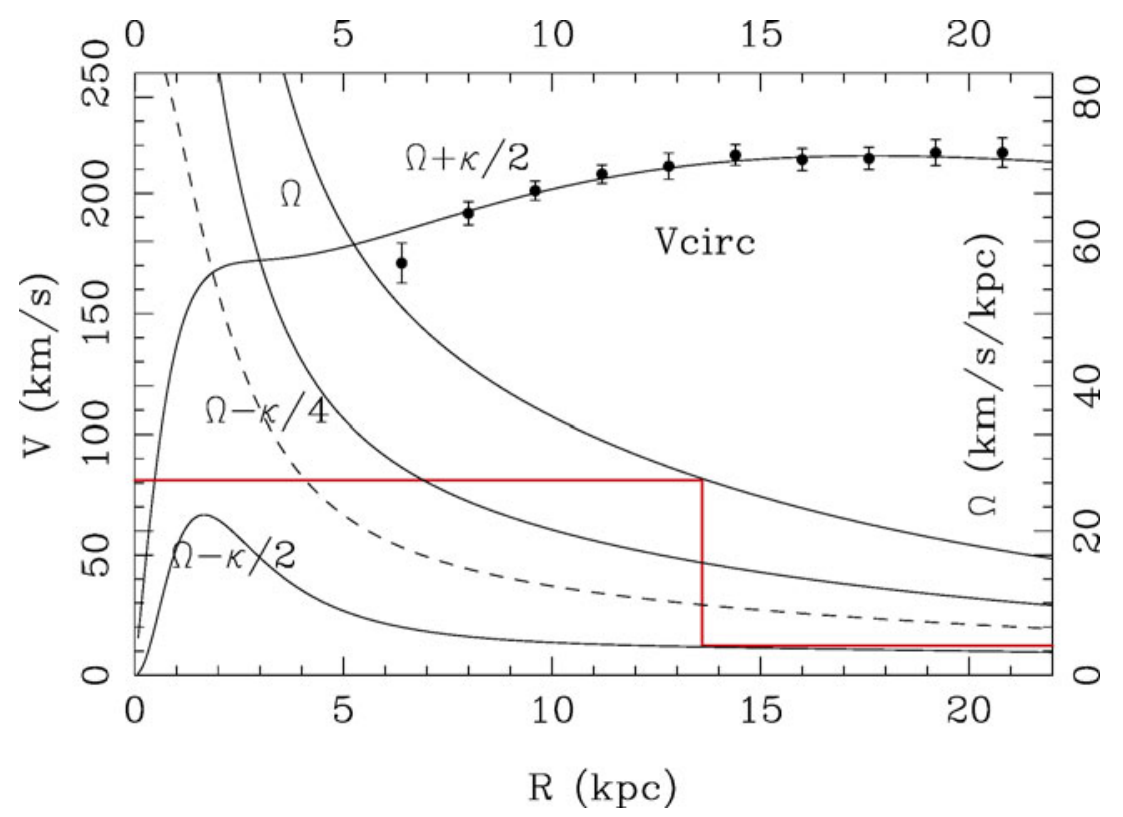

Figure 2. The rotational velocity in NGC 7479 (left-scale), and corresponding frequencies (right scale). The data points are from the HI kinematics (Laine et al. 1998a), and the model circular velocity has been fitted to them. In the central part, where the CO gas has strong non-circular motions, the model comes from the gravitational potential derived from the near-infrared image (Block et al. 2002). The central pattern speed of $27 \mathrm{~km} / \mathrm{s} / \mathrm{kpc}$ has been estimated by Laine et al. (1998b). The optical spiral pattern is outside corotation, and winds up at OLR. We propose here that this ring, which corresponds also to an HI ring, is the ILR of the external spiral pattern, with speed $4 \mathrm{~km} / \mathrm{s} / \mathrm{kpc}$.

the AM at the bar formation. When the gas inflows, it gives back AM to the bar, and weakens it, the mechanism can be called self-destruction of the bar.

The actual effect of the gas depends on the cooling, and is very different when the gas is considered isothermal or adiabatic (Debattista et al. 2006). In presence of cooling, the gas is very dissipative, which enhances its phase-shifted reponse and the amplitude of the torques: the bar destruction is then quite rapid.

Let us note that the AM exchange redistributes mass radially, and can explain exponential profiles. The succession of several bars could have signatures in density profiles breaks, that are now currently observed in may be $75 \%$ of galaxies (Pohlen 2002).

The bar destruction in presence of large gas fraction has also been seen in simulations of galaxy formation in a cosmological context, even in presence of massive triaxial dark matter haloes (Heller et al. 2007b). Bars form and drive gas inflows towards the center, which trigger formation of nuclear bars; this in turn weakens and destroys the primary bars. The amplitude of the $m=2$ component either in the stellar or gaseous component has a complex evolution (see Fig 3).

Bars can reform in galactic disks, through external gas accretion, and secular evolution. But the inflow of external gas has to wait the weakening of bars and their corresponding positive torques outside corotation, which prevent the gas enter. In that sense, gas accretion is intermittent; on a time-scale of several hundreds million years, the gas is first confined outside OLR until the bar weakens, then it can replenish the disk, to make it unstable again to bar formation. The gas inflow towards the very center, to feed AGN, 


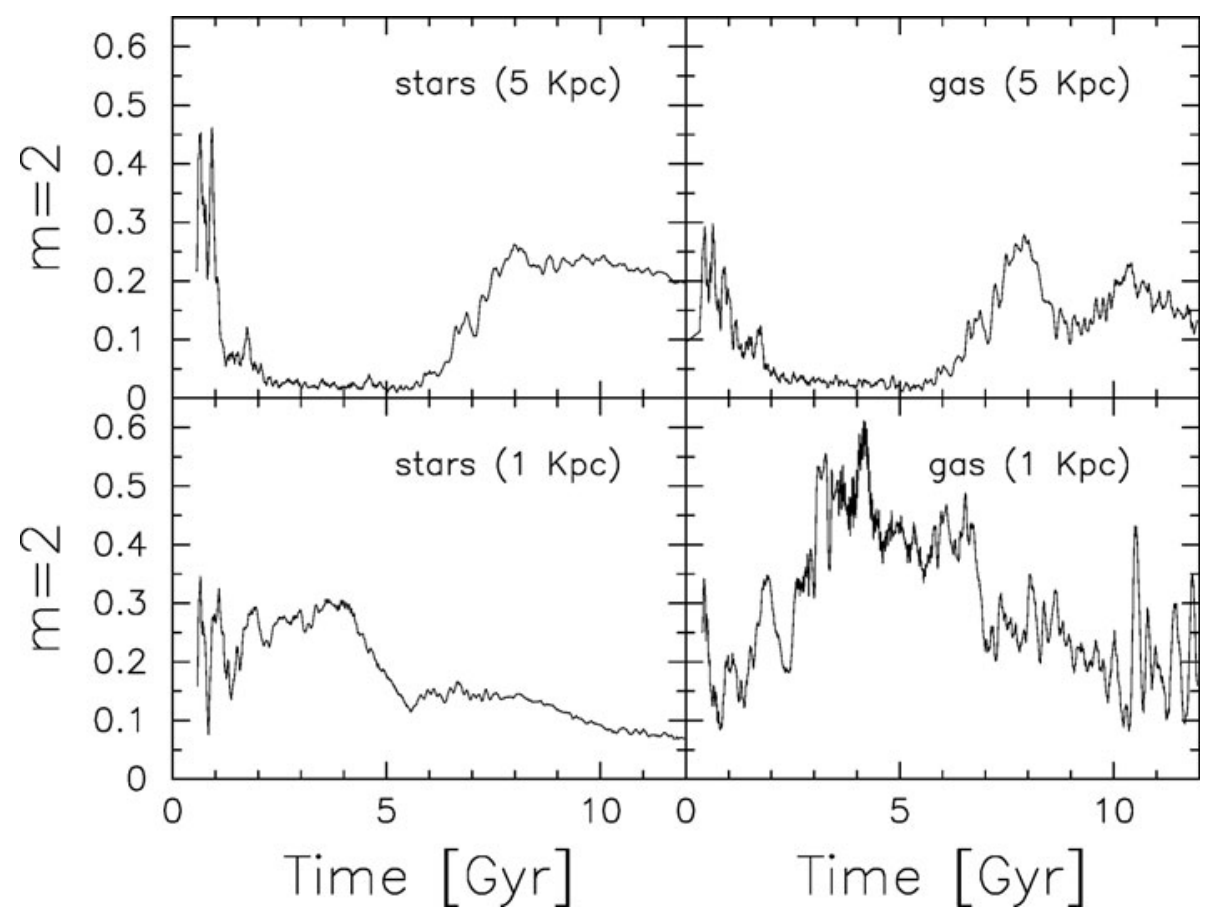

Figure 3. The evolution of a galaxy disk, inside a triaxial dark matter halo collapsed at $z=2$. The $m=2$ Fourier component is shown versus time for stars (left frames) and gas (right frames) within the central $5 \mathrm{kpc}$ (upper frames) and $1 \mathrm{kpc}$ (lower), from Heller et al. (2007b).

is also intermittent; the gas in a first phase is stalled at the ILR, and has to wait for the decoupling of a nuclear bar, to be driven further inwards. This final steps are quite rapid, of the order of the dynamical time-scales at these radii, which are of the order of 10 Myr.

Different conclusions have been drawn recently by Berentzen et al. (2007), with simulations of galaxies embedded in massive dark haloes. They compute the exchange of AM between stars and gas, and find it negligible, the AM exchange being essentially with the dark matter halo particles. Their gas fraction is always smaller than $8 \%$, may be insufficient for the gas to have a significant role in the AM transfer in presence of massive haloes.

However, they find that bars weaken and destroy more quickly in gas-rich galaxy disks, than in gas-poor ones, the separating gas fraction being $3 \%$. They interprete this phenomenon in terms of central mass concentration. The gas driven inwards by the bar creates a central mass concentration, which weakens the bar, in destroying the resonances and heating the stars. In gas poor galaxies, the bar lasts longer, and the main weakening mechanism for the bar is the peanut formation, through vertical resonances. Gas-rich galaxies have little or no peanut instability, because the central mass concentration damps it. One common conclusion, nevertheless, is that bars disappear more quickly in presence of gas.

The precise role of the dark matter halo can be quantified, by comparing for instance the dynamics of galaxies, treated with DM or without, but in modified gravity, so that they have the same rotation curves (e.g. Tiret \& Combes 2007). With a dark matter halo, the bar appears later, the disk being stabilised by it. In the MOND case, the disk is entirely self-gravitating and more unstable. For pure stellar disks without gas, the 

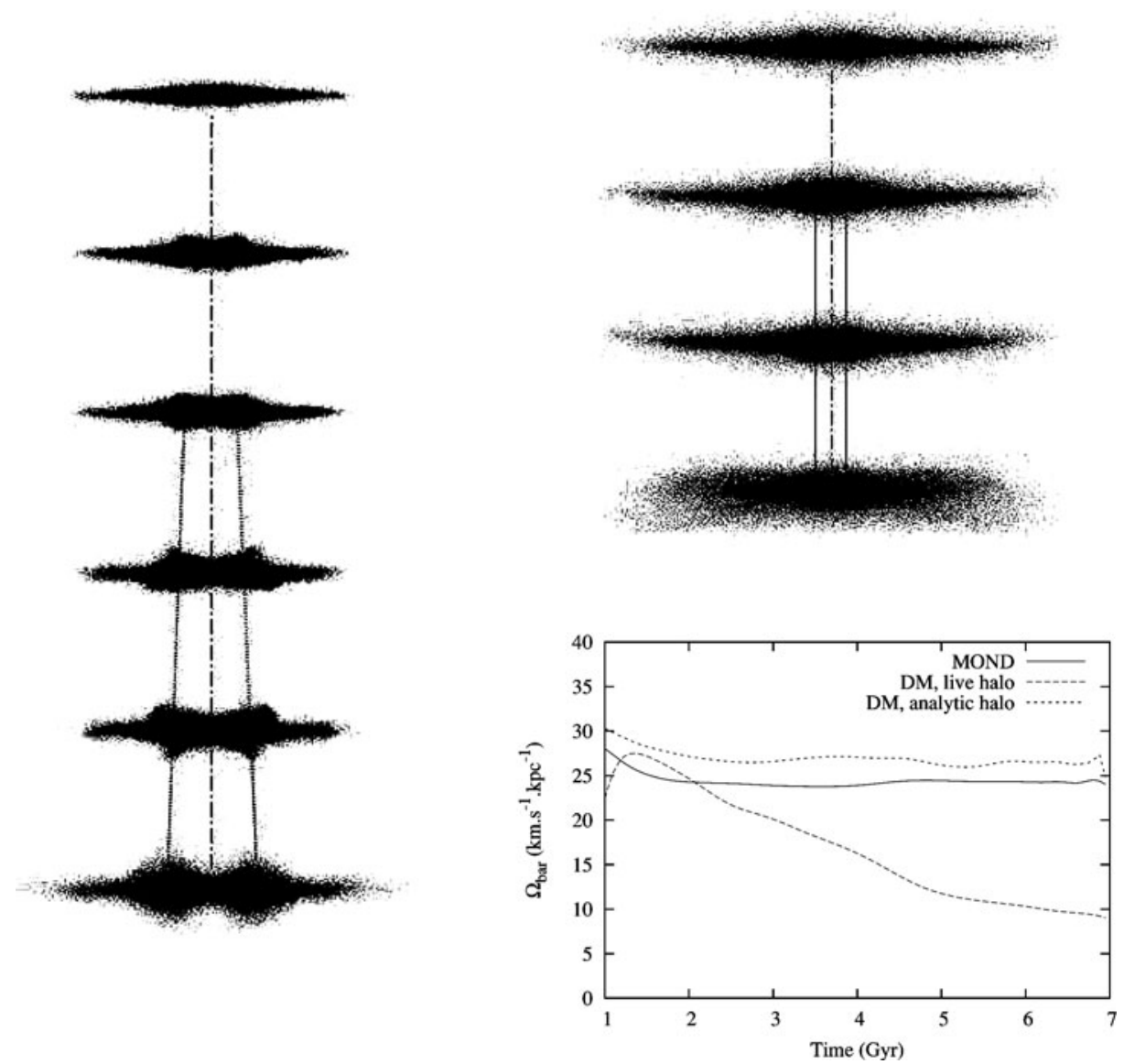

Figure 4. Left: peanut formation in a galaxy embedded in a dark matter halo, note the increase of the peanut radius with time. Top right: buckling instability in a MOND galaxy, the peanut radius is now fixed. Bottom right: Evolution with time of the bar pattern speed, in the case of a live dark matter halo, an analytical halo, or MOND. In both latter cases, the bar does not experience dynamical friction, and keeps a constant speed, from Tiret \& Combes (2007).

destruction of the bar occurs when the peanut instability thickens the disk vertically. The buckling instability occurs in both cases, but later in MOND. The main differences between the two models is the existence of the dynamical friction against the dark matter halo, and the exchange of angular momentum. Because of the friction, the pattern speed declines in the DM case, while it keeps constant in MOND (cf Figure 4). The resonance shifts in radius, and the peanut instability also. Through AM exchange with the halo, the bar can reform after the peanut weakening.

As for the role of gas, it has been shown also in cosmological simulations, that the bar strength declines faster in the presence of gas, but this is more visible when the dark matter halo is not dominating. The fraction of gas able to destroy the bar depends on the $\mathrm{DM} /$ disk mass ratio, it is $\mathrm{f}_{\text {gas }}=0.2$, when DM/disk $=3$ (Curir et al. 2007, see Figure 5). When the DM halo mass is negligible within the disk, this fraction falls down to $6 \%$.

Heller et al. (2007a) have investigated the formation of nested bars in the early evolution. The secondary bar lasts only 1-2 Gyr, and is destroyed after a large gas inflow to the center. In the same time, the primary bar weakens. 


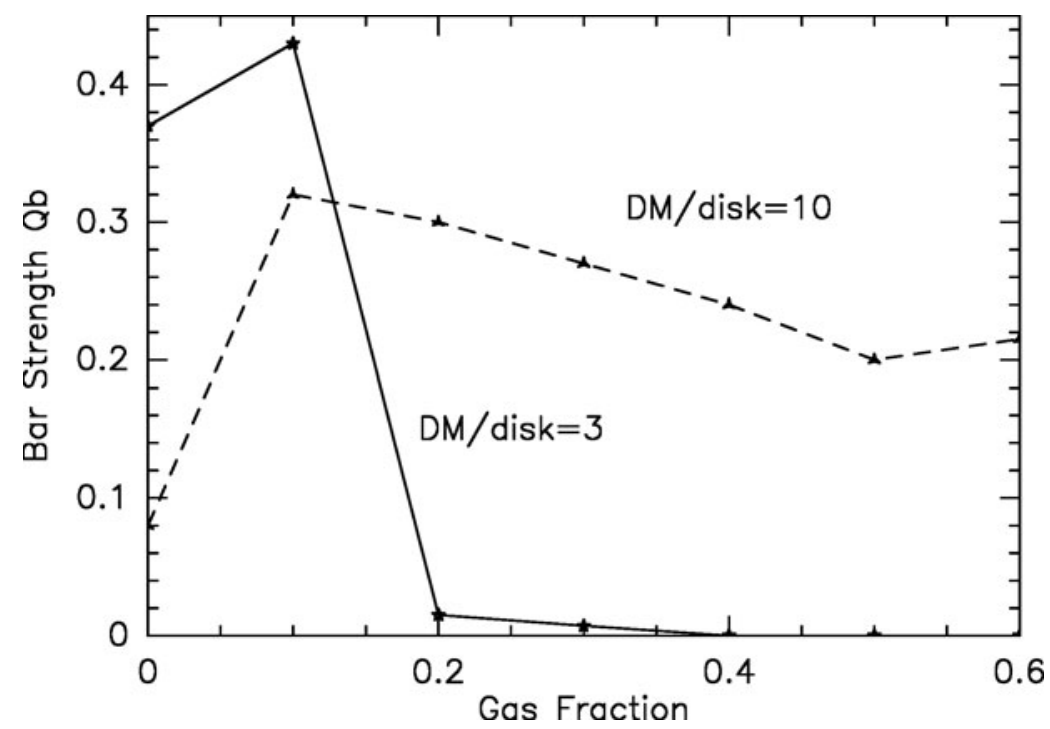

Figure 5. Bar strength $\mathrm{Q}_{b}$ of cosmologically evolved galaxies, versus their gas fraction, for two mass ratios between dark matter halo and baryonic disk: DM/disk $=3$ (full line) and 10 (dash), from Curir et al. (2007).

The dark matter halo plays a large role in the development of bar instability, and therefore gas flows in galaxy disks, but not only through its total mass, also through its radial profile. Figure 6 shows the gas distribution in disks embedded in DM haloes of the same mass, but one has an NFW profile, while the other is a Plummer. In the NFW case, the DM in the center is cuspy, which creates an axisymmetric mass concentration, and dilutes the gravity torques of the bar: the gas flows are hampered, while in the Plummer case, the gas flows towards the center, and develops the classical ringed/bar morphology.

\section{Cold gas accretion}

The majority of galaxies are barred (75\%) and most of them (80\%) are strongly barred, as found in the near infrared (Eskridge et al. 2002, Block et al. 2002). There is in particular a paucity of weak bars (Marinova \& Jogee 2007). The bar frequency appears constant with redshift (Jogee et al. 2005). Since bars are not long-lived in gaseous disks, the observed bar frequency in late-type galaxies require that secular evolution reform bars. This can be done through external gas accretion, which replenishes the disk and re-creates the conditions of bar instability, but not with galaxy interactions, which tend to heat and destroy thin disks.

Cold gas accretion can also explain the large frequency of lopsided galaxies, and in particular in late-types (77\% are asymmetric, Matthews et al. 1998). Even in the nearinfrared, about $20 \%$ of galaxies have their Fourier component $\mathrm{A}_{1}>0.2$. Lopsidedness can be produced in interactions, but is not long-lived, and an external mechanism is required (Bournaud et al. 2005b). Asymmetric gas accretion (with $\sim 4 \mathrm{M}_{\odot} / \mathrm{yr}$ ) can explain the observed frequency of $m=1$ perturbations and their long life-time.

Cold gas accretion is also required to reform disks after mergers and also to account for star formation histories that are not falling off exponentially with time, in intermediatetype spirals. Cosmological accretion can typically double the mass of a galaxy in 10 Gyr. Cold gas accretion helps to solve the angular momentum problem (D'Onghia et al. 2006). 


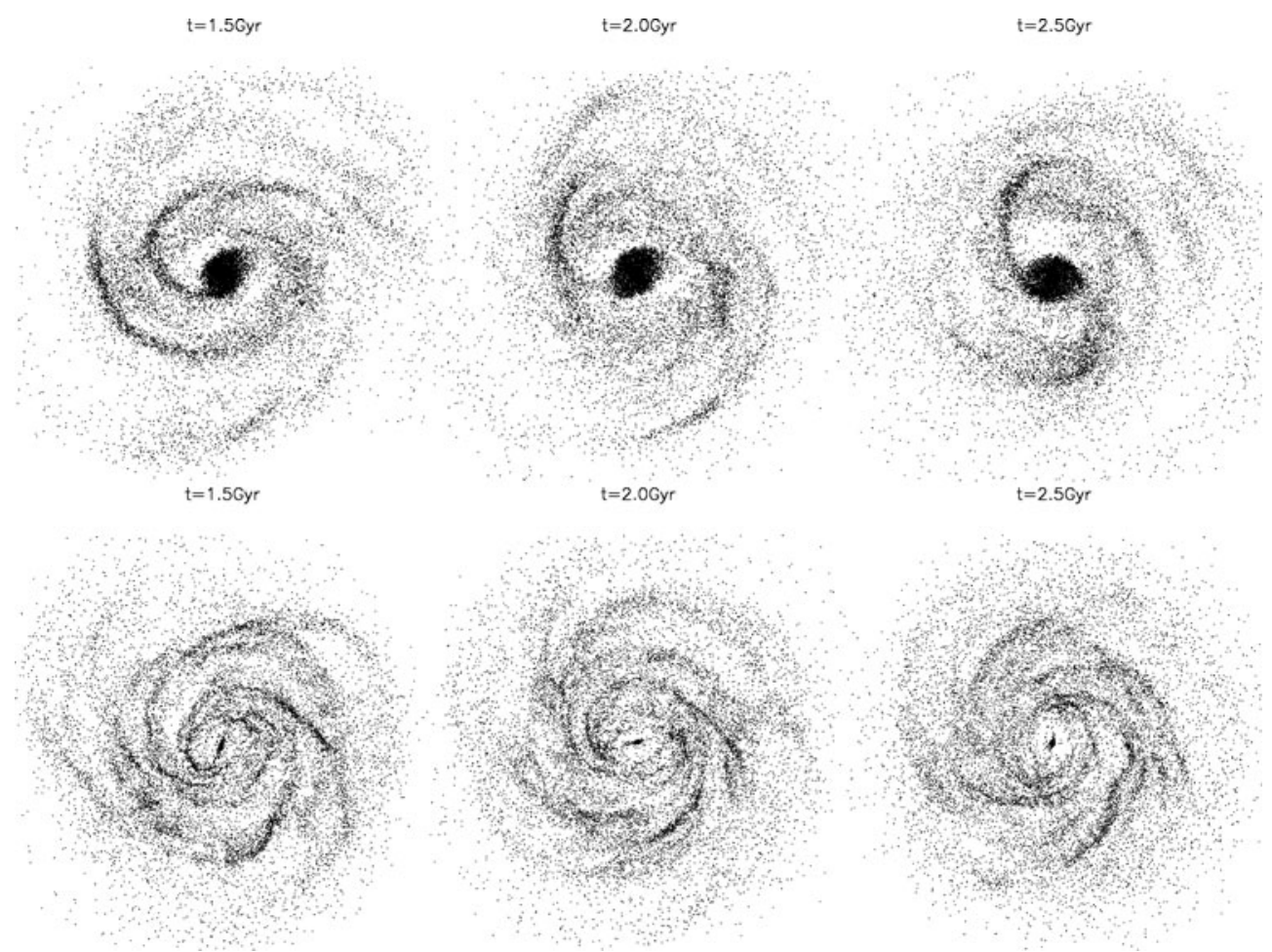

Figure 6. The evolution of the gas component depends on the shape of the dark matter halo, for the same total masss. Top: simulation in an NFW dark matter live halo, compared to Bottom: in a Plummer live halo. The simulations have been done with a Tree-SPH code, described in di Matteo et al. (2007a).

\section{AGN fueling and feedback}

Gas radial inflows are part of a dynamical cycle involving bars, they can feed AGN or starburst, that are themselves providing negative feedback to stop their activity. Gas flows are then intermittent. The time-scale corresponding to each phase can be estimated from the statistical frequency of galaxy observations in the precise phase.

From a detailed study of the molecular gas in the circumnuclear regions of active galaxies (NUGA project), it appears that the AGN fueling phase must be very short, corresponding to the dynamical time-scale in the central 100-200pc. In the very center, the gas is sometimes driven inwards through nuclear spirals, conspicuous from their dust absorption, but also underlined by $\mathrm{CO}$ emission. These nuclear spirals could be driven by non-axisymmetric patterns or by orbiting black holes (Etherington \& Maciejewski 2007).

Once the AGN activity is started, the energy released, and in particular the radio jets, provide a negative feedback to stop further gas accretion. The AGN feedback is however able to influence the galaxy as a whole only for large masses, and in particular in massive galaxies in the center of cooling flows. It can explain the exponential cut-off at the bright end of the galaxy luminosity function (Croton et al. 2006).

The exploitation of this feedback mechanism in cosmological simulations, allows to understand better the correlation and concomittant growth of spheroids and black holes, the AGN activity influencing and moderating the star formation activity of their hosts. Following their growth as a function of redshifts and along the merger hierarchy, it can be found that most of supermassive black holes accrete their mass in the quasar regime, 
driven by mergers. The quietest way (much lower than the Eddington rate) is marginal (Sijacki et al. 2007).

\section{Gas flows in interactions and mergers}

Important gas flows are triggered by galaxy interactions and mergers. They are also driven by gravity torques, produced by the non-axisymmetry of the stellar component, through bars essentially.

A recent series of merger simulations, varying the geometry of the galaxy interactions, the morphological types and mass ratios, has shown that gas inflows, and then the corresponding starbursts, were more efficient for retrograde encounters (di Matteo et al. 2007a). This might appear paradoxical, but can be explained when the sense of gas flows are computed in detail. In direct interactions, the tides are more resonant, and gas is efficiently driven outwards, in tidal tails. In retrograde encounters, on the contrary, the gas remains in the disks, and is inflowing due to bars.

The angular momentum exchanges are from the initial relative orbit to the dark matter halo essentially, but also to the visible disk, gas or stars. Some cases of retrograde encounters between an elliptical and a spiral galaxies, reveal the formation of counter-rotating cores, by a new mechanism (di Matteo et al. 2007b). Since tidal forces are stronger in the outer parts, the disk of the spiral is highly perturbed, and absorbs a large part of the negative relative orbital momentum. Its center is unaffected, and keeps its initial spin orientation. The stellar component from the elliptical, that was not rotating initially, also absorbs part of the orbital AM, as the dark matter. The counter-rotating effect is opposite to the mechanism described in Balcells \& Gonzalez (1998).

\section{Conclusion}

Secular evolution plays a fundamental role, in the fueling of starbursts and AGN, and in bulge formation. The bar instability is the main motor of this evolution, and is part of a dynamical cycle, leading to its own destruction. Starting a new cycle and reforming a bar requires diffuse cold gas accretion, from cosmic filaments, replenishing the galactic disk in gas.

Along the cycle, the stars in the disk exchange angular momentum with dark matter haloes or with the outer disk, or with gas, according to dark-to-visible mass ratio, saturation or resonances. The gas inflow itself leads to the decline in bar strength. The gas fraction able to destroy bars depend on the DM/disk mass ratio.

In addition to the total amount of dark matter halo, gas flows and bar strength depend on dark halo radial profiles, orbital chaos and resonances, gas fraction, vertical feedback (peanut), etc..

Bars trigger gas flows in galaxy interactions and mergers. The gas inflows and the subsequent starbursts are more efficient with retrograde orbits, since the tidal tails dragging the gas out of the galaxies are then less developped.

\section{References}

Athanassoula, E. 2002, ApJ 569, L83

Athanassoula, E. 2003, MNRAS 341, 1179

Athanassoula, E., Lambert, J. C., \& Dehnen, W. 2005, MNRAS 363, 496

Balcells, M. \& Gonzalez, A. 1998 ApJ 505, L109

Berentzen I., Shlosman, I., Martinez-Valpuesta, I., \& Heller, C. 2007, ApJ 666, 189 
Block, D. L., Bournaud, F., Combes, F., Puerari, I., \& Buta, R. 2002 A\&A 394, L35

Bournaud, F. \& Combes F. 2002, A\&A 392, 83

Bournaud, F., Combes F., \& Semelin B. 2005a, MNRAS 364, L18

Bournaud, F., Combes, F., Jog, C. J., \& Puerari, I. 2005b, A\&A 438, 507

Buta, R. \& Combes, F. 1996, Fundamentals of Cosmic Physics, Volume 17, pp. 95-281

Croton, D. J., Springel, V., White, S. D. M. et al. 2006 MNRAS 365, 11

Curir, A., Mazzei, P., \& Murante, G. 2007A\&A 467, 509

Debattista, V. P. \& Sellwood J. 2000, ApJ 543, 704

Debattista, V. P., Mayer, L., Carollo, C. M. et al. 2006, ApJ 645, 209

di Matteo, P., Combes F., Melchior A -L., \& Semelin B. 2007a, A\&A 468, 61

di Matteo, P., Combes F., Melchior A -L., \& Semelin B. 2007b, A\&A submitted

D'Onghia, E., Burkert, A., Murante, G., \& Khochfar, S. 2006, MNRAS, 372, 1525

D'Onghia, E. \& Navarro, J. F. 2007, MNRAS, in press

Eskridge, P. B., Frogel, J. A., Pogge, R. W. et al. 2002 ApJS 143, 73

Etherington, J. \& Maciejewski, W. 2006 MNRAS 367, 1003

Fisher D. B. 2006 ApJ 642, L17

Friedli, D. \& Benz W. 1993, A\&A 268, 65

García-Burillo, S., Combes, F., Schinnerer, E., Boone, F., \& Hunt, L. K. 2005, A\&A, 441, 1011

Heller, C. H., Shlosman, I., \& Athanassoula, E. 2007a, ApJ 657, L65

Heller, C. H., Shlosman, I., \& Athanassoula, E. 2007b, astro-ph/0706.3895

Jogee, S., Scoville, N., \& Kenney, J. D. P. 2005, ApJ 630, 837

Laine, S. \& Gottesman, S. T. 1998a, MNRAS 297, 1041

Laine, S., Shlosman, I., \& Heller, C. H., 1998b, MNRAS, 297, 1052

Laine, S., Kenney, J. D. P., Yun, M. S., \& Gottesman, S. T. 1999 ApJ 511, 709

Marinova, I. \& Jogee, S. 2007, ApJ 659, 1176

Matthews, L. D., van Driel, W., \& Gallagher, J. S. 1998, AJ, 116, 2196

Pohlen M. 2002, PhD Thesis, Ruhr-Universität, Bochum, Germany

Regan, M. W., Thornley, M. D., Vogel, S. N. et al. 2006, ApJ 652, 1112

Sakamoto, K., Okumura, S. K., Ishizuki, S., \& Scoville, N. Z. 1999, ApJ 525, 691

Shen J. \& Sellwood J. A. 2004 ApJ 604, 614

Sheth, K., Vogel, S. N., Regan, M. W. et al. 2005 ApJ 632, 217

Sijacki, D., Springel, V., di Matteo, T., \& Hernquist, L. 2007, astro-ph/0705.2238

Tiret, O. \& Combes, F. 2007, A\&A 464, 517 